\title{
DIFFEOMORPHIC COMPLETE INTERSECTIONS WITH DIFFERENT MULTIDEGREES
}

BY ANATOLY S. LIBGOBER AND JOHN W. WOOD 1

A complete intersection $X_{n}(\mathrm{~d}) \subset C P_{n+r}$ of hypersurfaces of degrees $d_{1}, \ldots, d_{r}$ is determined up to diffeomorphism by the dimension $n$ and the multidegree which is the unordered $r$-tuple $\mathbf{d}=\left(d_{1}, \ldots, d_{r}\right)$. A general problem is to find invariants which determine the diffeomorphism type of $X$. In this note we announce partial results which allow us to prove that in any odd dimension there are infinitely many pairs of multidegrees for which the corresponding complete intersections are diffeomorphic. This follows from a result characterizing the homotopy type of $X$ under some restrictions, an estimation of the number of differential structures with prescribed Pontryagin classes on a given homotopy type, and a counting argument. Complete proofs of these results will appear elsewhere.

A necessary feature of these examples is a large codimension.

Proposition. If $2 r \leqslant n$ and $n>2$, all complete intersections diffeomorphic to a given complete intersection of codimension $r$ have the same multidegree, where $\left(d_{1}, \ldots, d_{r}\right)$ with each $d_{i}>1$ may be identified with $\left(d_{1}, \ldots, d_{r}, 1, \ldots, 1\right)$.

Let $d$ be the total degree, $d=d_{1} \ldots d_{r}$, and assume $d= \pm 1 \bmod 8$. Let $n=2 m+1$. Then there is a differentiable connected sum decomposition $X_{n}$ (d) $=M_{n}(\mathrm{~d}) \# S^{n} \times S^{n} \# \cdots \# S^{n} \times S^{n}$ where $M_{n}(\mathrm{~d})$ has the homology of $C P_{n}$; this follows from a computation of the Kervaire invariant, see [5]. The cohomology ring structure of $M_{n}(\mathrm{~d})$ is $Z[x, y] /\left\{x^{m+1}=d y, x^{n+1}=0\right\}$ where the dimensions of $x$ and $y$ are 2 and $n+1$ respectively. We call a simply connected, $2 n$-dimensional $C W$-space $M$ with such a ring structure a $d$-twisted homology $C P_{n}$.

THEOREM 1. If $n=2 m+1$ and $d$ has no divisors less than $m+2$, then any two $2 n$-dimensional, $d$-twisted homology $C P_{n}$ 's are homotopy equivalent.

As a consequence, with these hypotheses the homotopy type of $X_{n}(\mathrm{~d})$ is determined by $n, d$, and the Euler characteristic. The theorem follows from a spectral sequence argument which shows $M$ is equivalent to the $2 n$-skeleton of the

Received by the editors December 27, 1979.

AMS (MOS) subject classifications (1970). Primary 14M10, 57D55.

1 The authors were partially supported by the National Science Foundation. 
fibre of the map $C P_{n} \rightarrow K(Z / d, n+1)$ defined by the cohomology class $y \bmod d$.

Theorem 2. Let $M$ be a smooth, d-twisted homology $C P_{n}$. Assume d has no divisors less than $n+3$. Then the number of differentiable structures on $M$ with given Pontryagin classes does not exceed the order of the reduced stable cohomotopy group $\pi_{s}^{\circ}\left(C P_{n}\right)=\lim \left[S^{q} \wedge C P_{n}, S^{q}\right]$.

The proof is an application of surgery theory, see [1], [2]. By the surgery exact sequence, the set of homotopy smoothings of $M, h S(M)$, embeds in $[M, G / O]$. Also there is an exact sequence

$$
\pi_{s}^{\circ}(M) \rightarrow[M, G / O] \rightarrow K O(M) .
$$

The image of $[M, G / O]$ is contained in the free part of $K O(M)$ and consequently if $M_{1}$ and $M_{2}$ in $h S(M)$ have the same Pontryagin classes, then they differ by an element of $\pi_{s}^{\circ}(M)$. The assumption on divisors of $d$ implies $\pi_{s}^{\circ}(M)=\pi_{s}^{\circ}\left(C P_{n}\right)$.

To construct diffeomorphic complete intersections it suffices, for a fixed $d$ satisfying our hypotheses, to find more complete intersections with given middle Betti number and Pontryagin classes than the order of $\pi_{s}^{\circ}\left(C P_{n}\right)$. These invariants depend on the first $n$ symmetric functions $\sigma_{1}, \ldots, \sigma_{n}$ of the multidegree. A counting argument shows that, for sufficiently large codimension $r$, the number of distinct multidegrees corresponding to a fixed (sufficiently large) degree $d$ is greater, by an arbitrarily assigned factor, then the number of possible corresponding values of the vectors $\left(\sigma_{1}, \ldots, \sigma_{n}\right)$.

The examples promised by this general theory have very large degrees and Betti numbers. However classification theorems for low dimensional manifolds permit us to give examples for $n \leqslant 3$. For algebraic curves the classification of real 2-dimensional surfaces gives many examples. The first two examples are $X_{1}(1)=X_{1}(2)=S^{2}$ and $X_{1}(2,2)=X_{1}(3)=S^{1} \times S^{1}$. For surfaces, $X_{2}(2,2,2)$ $=X_{2}(3,2)=X_{2}(4)$ are diffeomorphic $K-3$ surfaces. The classification of simply connected 4-manifolds up to homotopy type [3, p. 103] yields examples of homotopy equivalences-but there the diffeomorphism question remains open. The first three of these further examples are:

\begin{tabular}{|c|c|c|c|c|c|}
\hline Multidegree & Degree & Signature & Betti number & $c_{1}$ & $\begin{array}{l}\text { intersection } \\
\text { form }\end{array}$ \\
\hline $5,2,2,2,2,2$ & 160 & -1920 & 5758 & -6 & even \\
\hline $6,5,3$ & 90 & $"$ & " & -8 & " \\
\hline $6,6,6,2,2,2,2$ & 3456 & -131328 & 639358 & -16 & $"$ \\
\hline $8,4,4,3,3,3$ & $"$ & $"$ & $"$ & $"$ & " \\
\hline $6,6,6,2,2,2,2,2$ & 6912 & -269568 & 1403134 & -17 & odd \\
\hline $8,4,4,3,3,3,2$ & $"$ & $"$ & $"$ & $"$ & " \\
\hline
\end{tabular}


Wall's classification theorem [4] for simply-connected 6-manifolds gives examples of diffeomorphic 3 -folds. The first case is $X_{3}(16,10,7,7,2,2,2)=$ $X_{3}(14,14,5,4,4,4)$, both of degree 62720 . The examples were found by computer search; we thank Neil Rickert for guidance in this.

\section{REFERENCES}

1. W. Browder, Surgery on simply-connected manifolds, Ergebnisse der Mathematik und ihrer Grenzgebiete, Band 65, Springer-Verlag, New York-Heidelberg, 1972.

2. G. Brumfiel, Differentiable $S^{1}$ actions on homotopy spheres, Univ. of Calif., Berkeley, 1968.(mimeographed).

3. J. Milnor and D. Husemoller, Symmetric bilinear forms, Springer, New York, 1973.

4. C. T. C. Wall, Classification problems in differentiable topology. V. On certain 6-manifolds, Invent. Math. 1 (1966), 355-374.

5. J. W. Wood, Complete intersections as branched covers and the Kervaire invariant, Math. Ann. 240 (1979), 223-230.

DEPARTMENT OF MATHEMATICS, UNIVERSITY OF ILLINOIS AT CHICAGO CIRCLE, CHICAGO, ILLINOIS 60680 